\title{
Tunable photon lifetime in photonic molecules: a concept for delaying an optical signal
}

\author{
Yury P. Rakovich, John J. Boland, and John F. Donegan \\ Departments of Physics and Chemistry, Trinity College, Dublin 2, Ireland
}

Received April 26, 2005; revised manuscript received May 18, 2005; accepted June 6, 2005

We experimentally and theoretically studied the photon lifetime spectral distribution in two coherently coupled spherical microcavities of 3-16 $\mu \mathrm{m}$ diameter forming a photonic molecule, which shows a multipeak narrowband modal structure resulting from lifting of the mode degeneracy with respect to the azimuthal quantum number. The results demonstrate the feasibility of photonic molecules as a basis for a multichannel, wavelength-tunable optical delay-line device, which can be used for delay times in the range 10 ps to 1 ns. (C) 2005 Optical Society of America

OCIS codes: $230.5750,350.3950,060.4230,230.3120$.

Modern optical communications involve the use of short optical pulses with picosecond duration and gigahertz repetition rates to transmit data on an optical network. Synchronization of optical pulse trains within circuits in the network is provided by an optical delay-line device. Designs for compact devices with delay times in the range of $1 \mathrm{ps}$ to $1 \mu \mathrm{s}$ are required that will integrate well with photonic circuits.

Recent studies of optical mode structure in small coupled spherical microcavities ${ }^{1-5}$ (photonic molecules, PMs) have led to the observation of a modified electromagnetic field distribution that differs significantly from what would be obtained for a single microcavity. Experimental $^{2,4}$ and theoretical ${ }^{1,3}$ investigations showed that, depending on the orientation of the PM, whispering gallery modes (WGMs) of a monosphere can be split into two broadened modes of lower quality factor $(Q)$. In analogy with the quantum mechanical formation of molecular electronic orbits, these modes have been called bonding $(\mathrm{BN})$ and antibonding (ABN) states. $^{3}$ More recent investigations of the internal field distribution in a PM revealed a complex distribution of photonic states within the $\mathrm{BN}$ and $\mathrm{ABN}$ modes ${ }^{3,5}$ and therefore pointed the way toward consideration of applications of coupled microspheres (MSs). In this Letter we experimentally and theoretically demonstrate an application of a PM for the storing and buffering of optical pulses. The theoretical possibility of controlling the group velocity of optical pulses in coupled-resonator structures was studied previously. ${ }^{6-9}$ Recently, the occurrence of a resonance with higher $Q$ in the system of two MSs interacting through waveguides, mimicking electromagnetically induced transparency, was predicted. ${ }^{9}$ In this Letter we discuss a new and alternative method based on lifting of the WGM degeneracy with respect to the azimuthal quantum number $m$ in a system with strong coupling of two MSs. Such a system of coupled microcavities is proposed as a versatile building block suitable for integration in communication circuits because of its the size and spectral and material compatibility with optical fiber. The focus of our work is the fine structure of the BN and $A B N$ modes of the PM, which can be observed for off-axis excitation geometry, and which our group has recently demonstrated. ${ }^{5}$ A key feature of this modal structure is much higher $Q$ factors and therefore longer photon lifetimes in the PM compared with that of the single MSs forming the PM, which allow controllable manipulation with spectral distribution of mode lifetimes.

Below, we discuss the structure of coupled optical modes of a PM formed by two MSs (average size $3 \mu \mathrm{m}$ ) with a thin shell of CdTe nanocrystals [Fig. 1(a)]. The samples in use were fabricated and studied as described elsewhere. ${ }^{5}$ The peaks in the spectra of the individual MSs [Fig. 1(a)] are WGMs with mode number $n=21$, as follows from calculations based on Mie theory. The resonances from the PM are shown as a solid curve, and one can clearly see a large number of narrow peaks arising from the TE and TM resonances of the the individual MSs. The origin of this fine structure of $\mathrm{BN}$ and $\mathrm{ABN}$ modes lies in the lifting of the mode degeneracy in the $\mathrm{PM}^{3,5}$ and therefore this multipeak structure would not be radically altered in scattering spectra, in stimulated Raman scattering, or in lasing in the same way that in a semiconductor laser the lasing does not in general alter the cavity resonances. The deconvolution of the

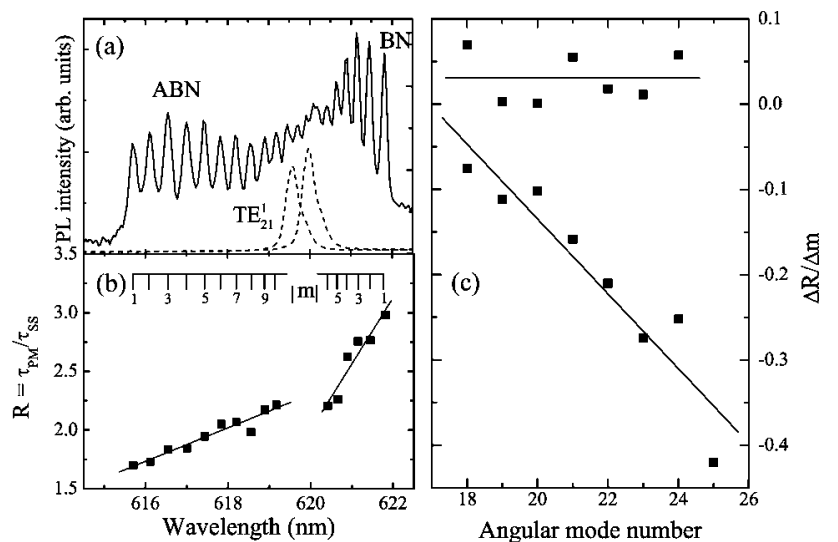

Fig. 1. (a) Photon lifetime spectra of the PM (solid curve) formed by two MSs with off-axis excitation and detection. Dashed curves, photon lifetime spectra of noninteracting MSs. (b) Ratio between the photon lifetime of $m$ modes of the PM and one of a single MS. (c) $n$ dependence of slopes $\Delta R / \Delta m$ for $\mathrm{BN}$ and $\mathrm{ABN}$ modes. 


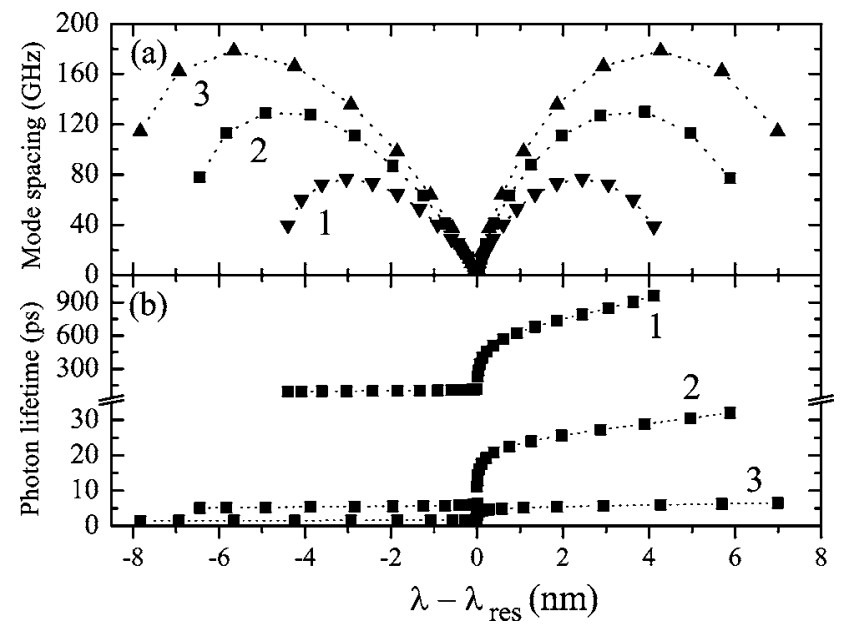

Fig. 2. (a) Spacing between $m$ modes calculated in the spectral region of the $C$ band for a PM formed by two identical MSs with sizes of (curve 3) $10 \mu \mathrm{m}$, (curve 2) $12 \mu \mathrm{m}$, and (curve 1) $16 \mu \mathrm{m}$. (b) Calculated distribution of the delay time between $m$ modes. The zero value on the wavelength axis corresponds to the position of $\mathrm{TE}_{24}, \mathrm{TE}_{29}$, and $\mathrm{TE}_{40}$ WGMs of the single MS.

line shape of the $m$ resonances of the PM by use of Lorentzian functions shows that the linewidths in the spectra of noninteracting MSs are much broader than the $m$ resonances of the PM [Fig. 1(b)]. Accordingly, the photon lifetime in the PM $\left(\tau_{\mathrm{PM}}\right)$ exceeds the photon lifetime in the single sphere before contact $\left(\tau_{\mathrm{SS}}\right)$. This experimental fact, along with estimated value of $\mathrm{BN}-\mathrm{ABN}$ splitting $(\sim 5-7 \mathrm{~nm})$, clearly suggests the possibility for development of a new PMbased photonic device such as an optical delay line with controllable spectral and temporal tunability.

In our experiments we have investigated strong coupling phenomena in the spectra of a PM in the visible and near-IR spectral regions. In order to study the feasibility of the PM as an optical delay device, we have analyzed the $m$-dependent structure for modes accommodated within the $C$ band from 1525 to $1565 \mathrm{~nm}$. For simplicity in our simulations we have considered only the case of identical MSs with WGMs having the radial quantum number $l=1$, taking into account only the interaction between WGMs of the same $n$. For a given WGM polarization, the value of the $\mathrm{BN}-\mathrm{ABN}$ splitting can be obtained from $^{3}$

$$
\Delta x_{m}=2 \Gamma_{n}\left|A_{n, m}\left(x_{n}\right)\right|,
$$

where $x_{n}=2 \pi R / \lambda_{n}$ is the size parameter of a resonance with mode number $n, \lambda_{n}$ is the corresponding resonant wavelength, and $\Gamma_{n}$ is the width of mode $n$ in a single MS. For a given radius of single MS $a$, the coefficient $A_{n, m}\left(x_{n}\right)$ can be calculated by using the single-mode tight-binding method, ${ }^{3}$ and the positions of the $m$ resonances can be estimated as $x_{m}$ $=x \pm \Delta x_{m} / 2$.

Figure 2(a) shows the values of spacing between adjacent $m$ resonances calculated in the spectral region of the $C$ band for PMs formed by two identical $\mathrm{SiO}_{2} \mathrm{MSs}$ with sizes of $10 \mu \mathrm{m}$ (curve 3), $12 \mu \mathrm{m}$ (curve
2 ), and $16 \mu \mathrm{m}$ (curve 1). In our model each $m$ resonance in the PM signal can be considered a channel, with spacing between channels depending on the size of the MS and the $m$ number. For $10 \mu \mathrm{m}$ individual MSs, only one TE mode $\left(\lambda_{24}=1542.4 \mathrm{~nm}\right)$ is found in the $C$ band. The maximum BN-ABN splitting (i.e., for $m=1$ ) of $15.7 \mathrm{~nm}$ was estimated in this case. Calculating the position of resonances for $16 \mu \mathrm{m}$ single MSs, we found two WGMs in the region of the $C$ band: $\mathrm{TE}_{40}(\lambda=1544.6 \mathrm{~nm})$ and $\mathrm{TM}_{39}(\lambda=1559.6 \mathrm{~nm})$. The splitting in this case is much smaller, $8.8 \mathrm{~nm}$ for the $\mathrm{TE}_{40}$ WGM, but accommodates a larger number of $m$ resonances. These results clearly demonstrate that two coupled MSs can generate a modal structure with a controllable number of peaks distributed across the major communications band, and the passband of the proposed delay-line device can be controlled by the size of the PM.

It is evident from Fig. 1(b) that the interaction between MSs results in periodic group-delay spectra with peaks occurring at each of the $m$ resonant frequencies, with longer delay times for higher $m$ values. To estimate the photon lifetimes in the PM in the $C$-band spectral region, we used the fact that the relative increase in photon storage time $R=\tau_{\mathrm{PM}} / \tau_{\mathrm{SS}}$ shows an almost linear dependence on $m$ number [Fig. 1(b)] for a given mode number $n$ over a wide spectral region $(n=17-25)$. It turns out that slope of this dependence $(\Delta R / \Delta m)$ in turn varies linearly with $n$ [Fig. 1(c)]. These experimental findings give us the possibility of extrapolating the $\Delta R / \Delta m$ dependence into a region of mode numbers $n$ that are characteristic of the $C$ band. The observed nonuniformity of spacing between $m$ resonances [Figs. 1 and 2(a)] together with a controllable number of modes provides a unique possibility of engineering the distribution of photon storage times in a desired spectral region. Figure 2(b) shows the estimated distribution of delay times between $m$ modes of the PM, taking values of the photon storage time in a single MS to be $\tau_{\mathrm{SS}}=55 \mathrm{ps}$ for $\mathrm{TE}_{40}^{1}, 3 \mathrm{ps}$ for $\mathrm{TE}_{29}^{1}$ and $0.9 \mathrm{ps}$ for $\mathrm{TE}_{24}^{1}$ WGMs. For the smallest PM size, $18 \mathrm{~m}$ modes are available within a window of $12-200 \mathrm{GHz}$ [Fig. 2(b)].

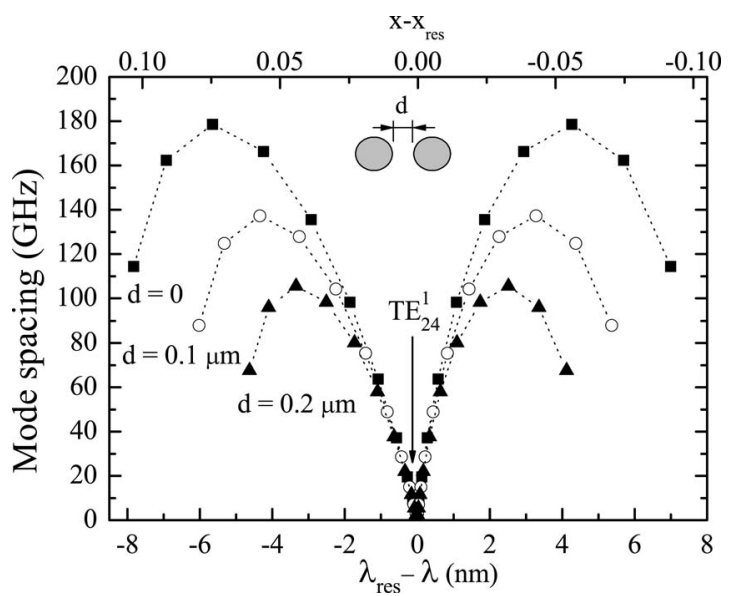

Fig. 3. Spacing between adjacent $m$ modes calculated for a PM formed by two identical MSs with a size of $10 \mu \mathrm{m}$ separated by distance $d$. 
The smaller $Q$ factor of the WGM in the MSs forming the PM results in a relatively moderate increase in delay time-from $1.5 \mathrm{ps}$ (obtained for the ABN resonance with $m=1$ ) to $7 \mathrm{ps}$ (calculated for the corresponding $m$ resonance of the BN mode of the PM). For MSs of larger size, the number of available modes increases. Indeed, for $12 \mu \mathrm{m}$ coupled MSs, 20 $m$ resonances fit into the indicated spacing window, with the biggest value of intermode spacing $\sim 130 \mathrm{GHz}$. Delay times distributed between these $m$ modes increase from 5.2 to $32 \mathrm{ps}$. The upper limit of intermode spacing drops even more for a PM formed from $16 \mu \mathrm{m}$ MSs. In that case, $22 \mathrm{~m}$ resonances with spacing between 12 and $76 \mathrm{GHz}$ can be seen in Fig. 2(a), providing discrete time delays, which are found to be distributed between 94.5 and 960 ps. This result shows that achieving tunable delays in the range from picoseconds to nanoseconds that are of great practical importance and which cannot be easily obtained with electromagnetically induced transparency are quite feasible with our PM structure. ${ }^{8,9}$

Based on theoretical considerations of the internal field in the $\mathrm{PM}^{3}$ and on experimental studies, ${ }^{2,4}$ it is clear that the size of the interacting MSs is not the only parameter that allows control of the spectral distribution of delay times. First, the efficiency of coupling between PM modes strongly depends on the spacing between the MSs, $d$. For the present values of MS sizes, mode numbers, and small $d$, an approximate equation for the coefficient $A_{n, m}\left(x_{n}, d\right)$ can be used. $^{3}$ Taking, for example, a PM formed from $10 \mu \mathrm{m}$ MSs, the maximum BN-ABN splitting shows a decrease by a factor 1.3 as the spacing between MSs increases from 0 to $0.1 \mu \mathrm{m}$. While the delay time and number of $m$ modes available between 12 and $200 \mathrm{GHz}$ are unaffected, the upper limit of mode spacings drops to $139 \mathrm{GHz}$ (Fig. 3). The next possibility for providing spectral tunability of photon storage times emerges from the strong dependence of splitting on the angle of incidence of the electromagnetic wave. $^{2,3,5}$ The PM spectrum was demonstrated to be much richer in detail for off-axis excitation. ${ }^{3,5}$ Finally, it remains to be noted that the parameters of mode coupling in the PM can also be governed by detuning the MS sizes. ${ }^{2}$

Using these results, we can draw a few general conclusions. Two coherently coupled MSs can generate modal structure with quality factors and photon lifetimes higher than that of single spheres. By analysing the $m$-dependent splitting of $\mathrm{BN}$ and $\mathrm{ABN}$ modes of PMs with size, we have introduced a new strategy for potential application of coupled spherical microcavities as multichannel, wavelength-tunable optical delay devices. In the proposed scheme the bandpass width, mode spacings, and delays depend only on the physical parameters of the interacting MSs and have the desired property of being independent of the modulation frequency of the communication signal.

This work was supported by Science Foundation Ireland under grant numbers 02/IN.1/I47 and 00/ PI.1/C077A.2 Y. P. Rakovich's e-mail address is Rakovicy@tcd.ie.

\section{References}

1. K. A. Fuller, Appl. Opt. 30, 4716 (1991).

2. T. Mukaiyama, K. Takeda, H. Miyazaki, Y. Jimba, and M. Kuwata-Gonokami, Phys. Rev. Lett. 82, 4623 (1999).

3. H. Miyazaki and Y. Jimba, Phys. Rev. B 62, 7976 (2000).

4. Y. Hara, T. Mukaiyama, K. Takeda, and M. KuwataGonokami, Opt. Lett. 28, 2437 (2003).

5. Y. P. Rakovich, J. F. Donegan, M. Gerlach, A. L. Bradley, T. M. Connolly, J. J. Boland, N. Gaponik, and A. Rogach, Phys. Rev. A 70, 051801 (2004).

6. G. Lenz, B. J. Eggleton, C. K. Madsen, and R. E. Slusher, IEEE J. Quantum Electron. 37, 525 (2001).

7. J. E. Heebner and R. W. Boyd, J. Mod. Opt. 48, 2629 (2002).

8. J. K. S. Poon, J. Scheuer, Y. Xu, and A. Yariv, J. Opt. Soc. Am. B 21, 1665 (2004).

9. L. Maleki, A. B. Matsko, A. A. Savchenkov, and V. S. Ilchenko, Opt. Lett. 29, 626 (2004). 